ARTICLE

DOI: $10.1038 / s 41467-018-06858-y$

\title{
Reconfigurable infrared hyperbolic metasurfaces using phase change materials
}

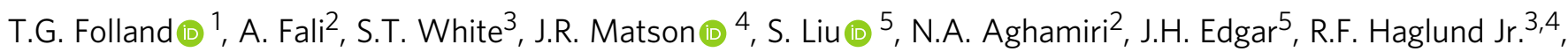
Y. Abate $^{2} \&$ J.D. Caldwell ${ }^{1}$

Metasurfaces control light propagation at the nanoscale for applications in both free-space and surface-confined geometries. However, dynamically changing the properties of metasurfaces can be a major challenge. Here we demonstrate a reconfigurable hyperbolic metasurface comprised of a heterostructure of isotopically enriched hexagonal boron nitride ( $\mathrm{hBN}$ ) in direct contact with the phase-change material (PCM) single-crystal vanadium dioxide $\left(\mathrm{VO}_{2}\right)$. Metallic and dielectric domains in $\mathrm{VO}_{2}$ provide spatially localized changes in the local dielectric environment, enabling launching, reflection, and transmission of hyperbolic phonon polaritons (HPhPs) at the PCM domain boundaries, and tuning the wavelength of HPhPs propagating in $\mathrm{hBN}$ over these domains by a factor of 1.6. We show that this system supports in-plane HPhP refraction, thus providing a prototype for a class of planar refractive optics. This approach offers reconfigurable control of in-plane HPhP propagation and exemplifies a generalizable framework based on combining hyperbolic media and PCMs to design optical functionality.

\footnotetext{
${ }^{1}$ Department of Mechanical Engineering, Vanderbilt University, Nashville, TN 37212, USA. ${ }^{2}$ Department of Physics and Astronomy, University of Georgia, Athens, GA 30602-2451, USA. ${ }^{3}$ Department of Physics and Astronomy, Vanderbilt University, Nashville, TN 37212, USA. ${ }^{4}$ Interdisciplinary Materials Science Program, Vanderbilt University, Nashville, TN 37212, USA. ${ }^{5}$ Department of Chemical Engineering, Kansas State University, Manhattan, KS 66506, USA. Correspondence and requests for materials should be addressed to Y.A. (email: yabate@physast.uga.edu)

or to J.D.C. (email: josh.caldwell@vanderbilt.edu)
} 
ptical near and far fields can be manipulated by scattering light into the resonant modes of nanostructured materials, which collectively form optical metasurfaces ${ }^{1-3}$. Historically, metallic polaritonic elements ${ }^{4}$ have been used, which are highly absorbing ${ }^{5}$ and typically exhibit limited tuning due to the geometric dependence of optical resonances. True reconfigurability - that is, complete changing of the optical responsetherefore becomes challenging, as it requires arbitrarily changing the shape of individual elements of the structure, dynamically controlling the local dielectric environment, or controlling the optical properties of the polaritonic material itself.

In this regard, phase-change materials (PCMs) offer an appealing approach to introducing true reconfigurability as they undergo significant changes in optical properties upon exposure to external stimuli6,7. Examples of PCMs are vanadium dioxide $\left(\mathrm{VO}_{2}\right)^{8-11}$ and germanium antimony telluride (GeSbTe) glasses $^{6,12}$, which undergo dielectric to metallic phase transitions upon heating or pulsed-laser excitation. For $\mathrm{VO}_{2}$, this is a volatile (non-latching) phase transition, whereas GeSbTe undergoes a non-volatile (latching) transition. By integrating PCMs and polaritonic materials, changes in optical properties induced by such a phase transition can provide the means to control the polariton dispersion by changing the local dielectric environment in which the evanescent polaritonic near-fields propagate. Thus, they can be exploited to realize reconfigurable metasurfaces ${ }^{6,12-17}$. However, one of the phases of PCMs is typically metallic and/or exhibits high optical losses. Consequently, in previous studies of surface-confined polaritons, such as surface plasmon or surface phonon polaritons, the propagation was restricted to spatial regions over the PCM where a low-loss dielectric phase was present $^{6,12}$. This makes concepts such as nanophotonic waveguides, grating couplers and focusing elements extremely difficult to realize in PCM-surface-polariton-based systems, despite the opportunities available.

Here we exploit two key changes in approach that overcome these previous limitations. First, we significantly reduce losses in polariton propagation by using isotopically enriched hexagonal boron nitride $\mathrm{e}^{18,19}(\mathrm{hBN})$, a natural hyperbolic ${ }^{20-24}$ medium that supports low-loss hyperbolic phonon polaritons (HPhPs). Second, by exploiting hyperbolic polaritons instead of the surfaceconfined variety ${ }^{6,12-16}$, the polaritons remain sensitive to local changes in the dielectric function of the ambient environment ${ }^{25}$, whereas the electromagnetic near-fields are strongly confined to the volume of the hyperbolic material ${ }^{20,21,26}$. This means that $\mathrm{HPhPs}$ can interact with spatially localized phase transitions of the PCM, yet do not suffer significant optical losses from this interaction, and thus should propagate over both metallic and dielectric phases. Crucially, we show this to be the case, and that the difference in the local dielectric environment between the metallic and dielectric domains results in a large change in the $\mathrm{HPhP}$ wavelength in the hBN over each domain, which in turn results in the refraction of the polariton when transmitting across the PCM phase-domain boundaries. This means that the combination of hyperbolic media and PCMs employed here can be used to create refractive optical elements and waveguides ${ }^{27}$, as well as components benefitting from full optical functionalities that to this point have been limited to far-field optics. We demonstrate such concepts using electromagnetic modeling, showing that PCM-HPhP heterostructures can be designed as optical resonators ${ }^{20,28}$ and metasurfaces ${ }^{29,30}$, as well as refractive near-field components, such as waveguides and lenses. This combination of PCMs with hyperbolic media opens a whole new toolset for near-field optical design and structuring. Significantly, for reversible PCM transitions, any of these designs can be fully reconfigured using either thermal changes or approaches based on laser writing. Finally, by exploiting the increasingly wide range of different PCMs and hyperbolic materials and metamaterials, such as transition metal oxides ${ }^{31}$, these effects can be realized over an extended range of frequencies.

\section{Results}

Near-field measurements of hyperbolic polaritons. The prototype device (Fig. 1a, b) consists of a $24 \mathrm{~nm}$-thick flake of ${ }^{10} \mathrm{~B}$ enriched hBN ( $99 \%$ enriched $\left.{ }^{18,19}\right)$ transferred using lowcontamination transfer techniques onto a single crystal of $\mathrm{VO}_{2}$ grown on quartz. We use scattering-type scanning near-field optical microscopy (s-SNOM) to directly map and visualize the evanescent optical fields on the structure, corresponding to polaritonic waves of compressed wavelength $\lambda_{\mathrm{p}}$, propagating primarily within the volume of the hBN slab (see Fig. 1a). In sSNOM images, HPhPs can be observed in two ways: first, polaritons launched by the light scattered from the s-SNOM tip propagate to and reflect back from sample boundaries (e.g., a flake edge) creating interference fringes with spacing $\lambda_{\mathrm{p}} / 2$, which are scattered back to free space by the tip and detected ${ }^{21,32,33}$. Alternatively, polaritons can be directly launched from sample edges and propagate across the surface to interfere with the incident field at the tip, producing fringes with spacing $\lambda_{\mathrm{p}} 18,34$ Thus, in s-SNOM maps, a superposition of both so-called "tiplaunched" and "edge-launched" fringes may be observed and are interpreted by considering the fringe spacing from individual waves $\left(\lambda_{\mathrm{p}} / 2\right.$ vs. $\left.\lambda_{\mathrm{p}}\right)$ and the direction of polariton propagation.

Controlling hyperbolic polaritons using a PCM. The presence of both tip- (wavelength $\lambda_{\mathrm{p}} / 2$, purple line in the $x$ direction) and edge-launched (wavelength $\lambda_{\mathrm{p}}$, blue line in the $y$ direction) HPhPs can be readily observed in the hBN (Fig. 1c) slab transferred on top of the $\mathrm{VO}_{2}$ single crystal. Here, this is visualized via the nearfield amplitude s-SNOM image collected using a $1450 \mathrm{~cm}^{-1}$ excitation laser at room temperature. The observation of both tipand edge-launched modes in the $x$ direction, whereas only edgelaunched modes being apparent along the $y$ direction derives from the properties of the boundaries in the heterostructure sample, namely the edges of the $\mathrm{hBN}$ and $\mathrm{VO}_{2}$ crystals. As in previous experiments ${ }^{18,21,34}$, the edge of the hBN crystal provides for both high reflection of tip-launched HPhPs as well as a sharp edge for direct initiation of edge-launched modes ( $x$ direction). In contrast, the small size (440 nm thickness, $6.5 \mu \mathrm{m}$ width) of the $\mathrm{VO}_{2}$ crystal provides sufficient momentum to robustly scatter into $\mathrm{HPhP}$ modes at the $\mathrm{VO}_{2}$ crystal edges (bottom/top edges in Fig. 1c $)^{18,34}$. However, the interface between $\mathrm{VO}_{2}$ and air at the crystal edge provides a significantly reduced reflection coefficient, which suppresses tip-launched waves, an effect observed in prior work $^{12,25}$. A more detailed discussion of the occurrence of both tip- and/or edge-launched modes in the s-SNOM images is available in Supplementary Note 1 and Supplementary Fig 1.

Propagation of HPhPs is strongly influenced by the local dielectric environment ${ }^{25,29}$, so we investigated the influence of the $\mathrm{VO}_{2}$ phase transition by measuring the s-SNOM response of the sample as a function of temperature, traversing the full dielectricto-metal transition from $60^{\circ} \mathrm{C}$ to $80^{\circ} \mathrm{C}^{11}$. The sample was heated in situ inside the s-SNOM microscope on a custom-built heating stage. Individual $\mathrm{VO}_{2}$ domains are directly observed with sSNOM due to the dielectric contrast between domains, with metallic (dielectric) $\mathrm{VO}_{2}$ appearing as bright (dark) regions (Fig. 1d $)^{8-11}$. As the device is heated further (Fig. 1e), the hBNsupported HPhPs are observed to propagate over both the metallic and dielectric domains of $\mathrm{VO}_{2}$, for appreciable propagation distances in both regions. This contrasts with an earlier work focused on surface polaritons and PCMs, where the polaritons propagated for only a few cycles over the dielectric phase and 
$\mathbf{a}$
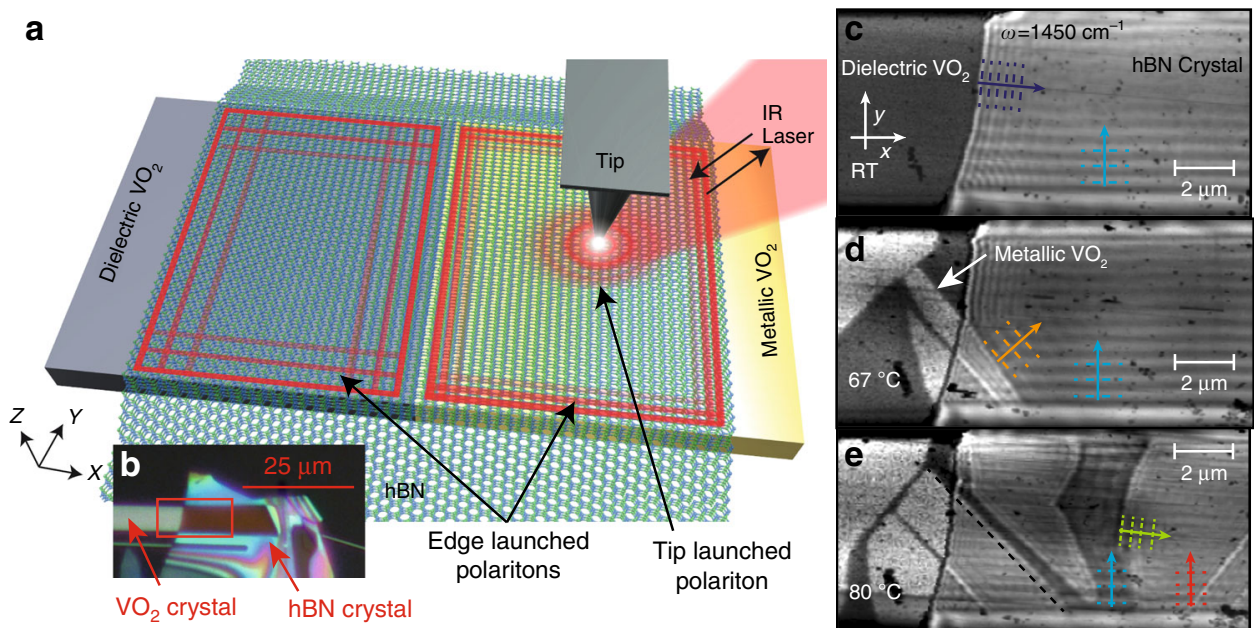

Fig. 1 Actively reconfigurable hyperbolic metasurface device. a A device and experimental schematic, in which hBN has been transferred on top of a $\mathrm{VO}_{2}$ single crystal and polaritons are imaged by the s-SNOM tip. b An optical microscope image of the heterostructure. c-e s-SNOM images of the optical nearfield at $1450 \mathrm{~cm}^{-1}(6.9 \mu \mathrm{m})$ at various temperatures, showing $\mathrm{HPhPs}$ propagating over both metallic and dielectric $\mathrm{VO}_{2}$ domains. The complex patterns that form are the consequence of multiple interfering waves over the different domains. The arrows show the following: purple highlights tip-launched modes reflected from the $\mathrm{hBN}$ edge, whereas blue designates the $\mathrm{HPhP}$ propagating within the interior of the $\mathrm{hBN}$ from the edge of the dielectric $\mathrm{VO}{ }_{2}$ crystal (boundary with air, suspended hBN). The red arrow highlights the same propagation characteristics as the blue arrow, except for HPhPs

propagating over the metallic $\mathrm{VO}_{2}$ domains. Finally, the orange and green arrows designate HPhPs propagating within the hBN from the domain boundaries between the dielectric and metallic domains of the $\mathrm{VO}_{2}$, with the orange (green) propagating over the dielectric (metallic) domains. In $\mathbf{e}$, the black dashed line indicates the edge of the $\mathrm{VO}_{2}$ metallic domain, extrapolated from the domain outside the $\mathrm{hBN}$ crystal

were entirely precluded from propagation over the metallic regions ${ }^{12}$. We attribute this difference to the volume confinement of the local electromagnetic near-fields of HPhPs supported within the low-loss $\mathrm{hBN}^{18,20,21}$, which prevents the polaritonic fields from being absorbed by the lossy metallic phase of $\mathrm{VO}_{2}$. After heating to high temperatures and performing these sSNOM measurements, allowing the device to cool to room temperature resets the $\mathrm{VO}_{2}$ crystal to its dielectric state, after which the sample can be reheated to get a different PCM domain pattern (see Supplementary Fig. 2). This allows us to reconfigure our device to study the propagation of HPhPs in a range of different geometries and at different frequencies within the same device. The large permittivity difference between metallic and insulating phases of $\mathrm{VO}_{2}$ therefore presents an excellent platform to manipulate and control polariton propagation within hyperbolic materials.

When s-SNOM maps the evanescent fields of propagating $\mathrm{HPhP}$ waves in the presence of multiple interfaces, complex images result from the superposition of the waves launched, transmitted across and reflected by each domain boundary, crystal edge, and the s-SNOM tip. The simplest polaritons to identify are the modes launched from the edge of the $\mathrm{VO}_{2}$ crystal, as they form straight fringes aligned parallel to the crystal edge. Due to the difference in local dielectric environment, these HPhPs possess different polariton wavelengths $\lambda_{\mathrm{p}}$ above each domain. Here the HPhP mode launched by the $\mathrm{VO}_{2}$ crystal edge over the dielectric (metallic) domain is highlighted by the light blue (red) arrow in Fig. 1c, d and demonstrate that the $\mathrm{HPhP}$ wavelength is modified from $\lambda / 12.9$ to $\lambda / 20.4$ by the PCM at 1450 $\mathrm{cm}^{-1}$ between these domains, serving as the first report of the dispersion of HPhPs being tuned by a PCM. Propagation lengths $(1 / e)$ are approximately $2.83 \mu \mathrm{m}$ (5.2 cycles) and $0.8 \mu \mathrm{m}(2.5$ cycles) in the dielectric and metallic phases at this frequency, respectively, which is comparable to propagation lengths in naturally abundant $\mathrm{hBN}(\sim 3.1$ and $2.5 \mu \mathrm{m}$ at the same wavevectors, respectively $)^{21}$. Furthermore, in Fig. 1d-e, sSNOM images show that HPhPs are directly launched in the
hBN over the boundaries between the dielectric (orange arrow) and metallic (green arrow) domains, despite there being no appreciable change in the topography of the $\mathrm{VO}_{2}$ crystal (Supplementary Note 2 and Supplementary Fig. 3). Although past work has shown that PCM domain boundaries can serve to launch polaritons ${ }^{12}$, here they are launched and propagated over both phases, with a different wavelength over each, promising the potential for dynamically reconfiguring $\mathrm{HPhP}$ properties and propagation. Note that the $\mathrm{VO}_{2}$ domains appear to change size when underneath the $\mathrm{hBN}$ (as seen by following the black dashed line in Fig. 1e). This arises from hyperlensing by the $\mathrm{hBN}^{35,36}$, which acts to magnify light scattered into a hyperbolic medium and can give rise to spatial regions on the edge of a domain where the wavelength appears not to change (seen to the left of the blue arrow in Fig. 1e).

Refraction of hyperbolic polaritons. This heterostructure also enables the transmission of polaritons across the aforementioned domain boundaries. To simplify s-SNOM images and subsequent analysis, domain geometries with only a single boundary are required. As the positions of domain boundaries induced via thermal cycling of the $\mathrm{VO}_{2}$ phase change are naturally quasirandom, we implemented multiple heating and cooling cycles (the same process as Supplementary Fig. 2) to achieve single dielectric-metal interfaces on the $\mathrm{VO}_{2}$ crystal for study. Examples are shown in Fig. 2a, b (also Supplementary Note 3 and Supplementary Fig. 4). Such "reconfiguring" of the metasurface has been repeated more than eight times in our experiments, with no appreciable change in the dielectric properties of either of the two phases of $\mathrm{VO}_{2}$ or the hBN flake, demonstrating the repeatability of this process.

Of particular interest is the polariton wave front that propagates away from the $\mathrm{VO}_{2}$ crystal edge in the $y$ direction (purple dashed line with black arrows in Fig. 2a, b): it meets the domain boundary and distorts, propagating in a direction that is not normal either to the domain or crystal edge. This is a 


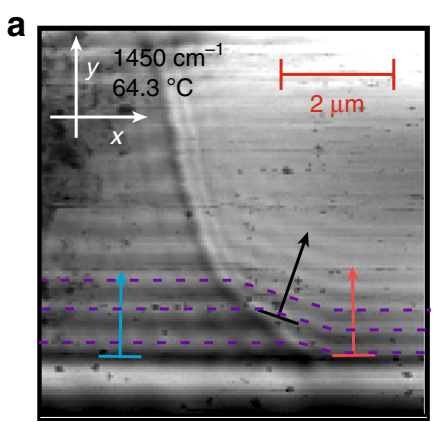

d

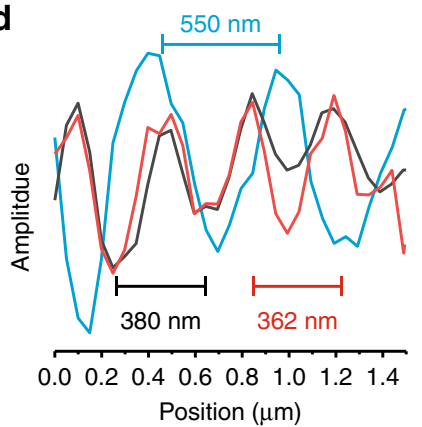

b

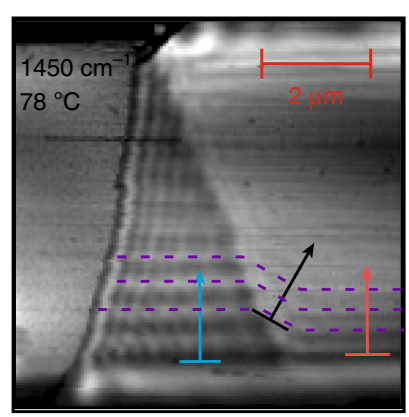

e

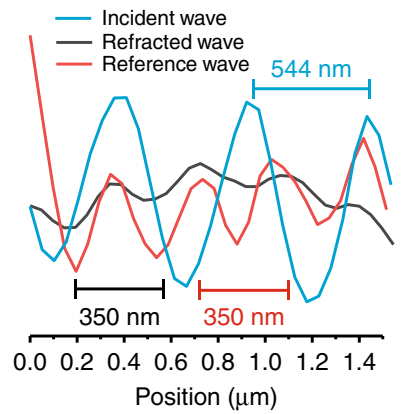

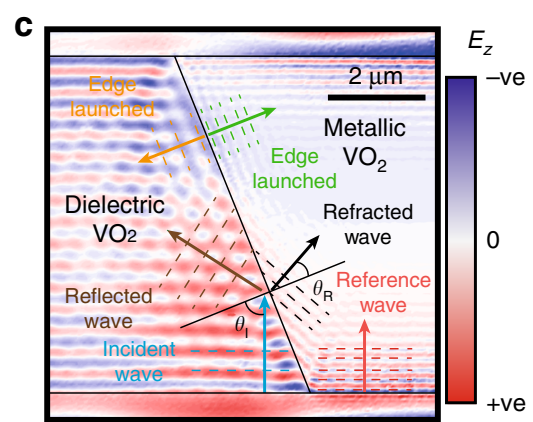

f

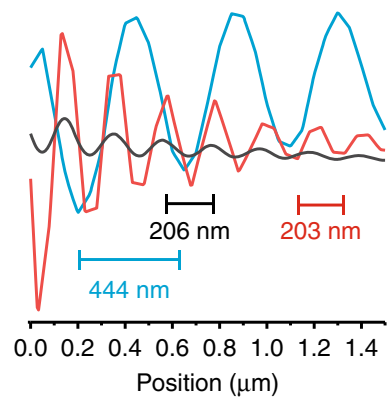

Fig. 2 Hyperbolic polariton refraction within a $\mathrm{hBN}-\mathrm{VO}_{2}$ heterostructure. $\mathbf{a}, \mathbf{b}$ Two s-SNOM maps of the near-field amplitude in the region of the domain boundary showing refraction. Purple dashed lines show the distorted phase front that propagates over the boundary. $\mathbf{c}$ An electromagnetic-field simulation of the geometry in $\mathbf{b}$, showing reflected, refracted and edge-launched waves. $\mathbf{d}$-f Line profiles from $\mathbf{a}$ to $\mathbf{c}$, respectively, showing refraction of the wave

signature of planar polariton refraction as the wave changes direction due to the local change in dielectric environment. Although planar polariton refraction has been reported previously for plasmon polaritons ${ }^{37}$, this is the first direct observation of refraction for hyperbolic polaritons and the first evidence for such polariton refraction as a function of incident (transmitted) polariton angle.

If a hyperbolic polariton traverses the boundary between metallic and insulating $\mathrm{VO}_{2}$ domains, the angle of propagation changes to conserve momentum in accordance with Snell's law: ${ }^{38}$

$$
\frac{\sin \left(\theta_{\mathrm{I}}\right)}{\sin \left(\theta_{\mathrm{R}}\right)}=\frac{n_{2}}{n_{1}}
$$

where $n_{1}$ and $n_{2}$ are the indices of refraction in the first and second media, and $\theta_{\mathrm{I}}$ and $\theta_{\mathrm{R}}$ are the corresponding angles of incidence and refraction. To demonstrate that the experimentally measured images are due to refraction, we compare the results in Fig. $2 \mathrm{~b}$ to a simplified electromagnetic simulation (Fig. $2 \mathrm{c}$ ). In the simulation, we excite the structure with plane waves $\left(45^{\circ}\right.$ incidence) and at the edges of the $\mathrm{VO}_{2}$ crystal and polaritonic waves are launched that propagate across the surface, mimicking edge-launched polaritons. Note that we ignore the tip-sample interaction in these simulations. Instead, $\mathrm{HPhPs}$ excited at the edge of the $\mathrm{VO}_{2}$ crystal (blue) propagate in the $y$ direction within the dielectric phase. When these HPhPs approach the angled dielectric-metallic domain boundary (black line), some of the wave will be reflected (brown) and some will be transmitted across the boundary (black) and refracted due to the mismatch in wavevectors for the HPhPs supported over the two PCM domains. The simulation also shows waves launched directly from the domain boundary (orange and green) in Fig. 1c, d. The refracted wave will not propagate normal to either the edge of the crystal or the domain boundary but will have the same polariton wavelength as the wave launched in the hBN by scattering of incident light from the metallic $\mathrm{VO}_{2}$ crystal edge. This is indeed what is shown in our experiments by the corresponding line profiles provided in Fig. 2d-f. However, the wave reflected by the metal-dielectric domain boundary is not observed experimentally due to interference with the edge-launched mode shown in light blue. Despite this, the good agreement between Fig. $2 b$ and $c$ shows clear evidence of $\mathrm{HPhP}$ refraction. Although in principle these effects should be observable also with a tip-launched waves in s-SNOM images, during our experiments however, we were unable to form a $\mathrm{VO}_{2}$ domain boundary sufficiently close to the flake edge (seen in Fig. 1b) to study this effect.

Quantifying polariton manipulation. To quantify the change in the polariton wavevector and $\mathrm{HPhP}$ refraction induced by the $\mathrm{VO}_{2}$ domains and to test the ability to reconfigure the metasurface, we systematically studied the polariton wavelength dependence on incident frequency and refracted angle in different domain geometries. In the first case, we systematically recorded sSNOM images at several monochromatic incident laser frequencies in both metallic and dielectric domains, and subsequently extracted the polariton wavelength through Fourier analysis (see Supplementary Note 3 and Supplementary Fig. 5) of the s-SNOM maps, as has been reported previously $18,21,32,33$. The experimentally extracted polariton wavevector (symbols) agrees well with numerical calculations of the HPhP dispersion for thin hBN slabs on a substrate consisting of either the dielectric or metallic phase of $\mathrm{VO}_{2}$ (Fig. 3a, b). In our assignment of the points in Fig. 3a, b, we consider both tip- and edge-launched modes, above both metallic and dielectric $\mathrm{VO}_{2}$, which can be observed in Supplementary Fig. 5. Again, this dramatic change in wavevector between domains at the same incident frequency is attributable to the large change in dielectric constant in $\mathrm{VO}_{2}$ between the two PCM states, which further compresses the polariton wavelength.

From the measured change in polariton wavelength, we calculated the ratio of the indices of refraction, $n_{1} / n_{2}$ to determine the expected angle of refraction for the HPhP waves from Eq. (1) and compared this with the refracted angle extracted from the sSNOM images in Fig. 2 and Supplementary Fig. 4, to test the 

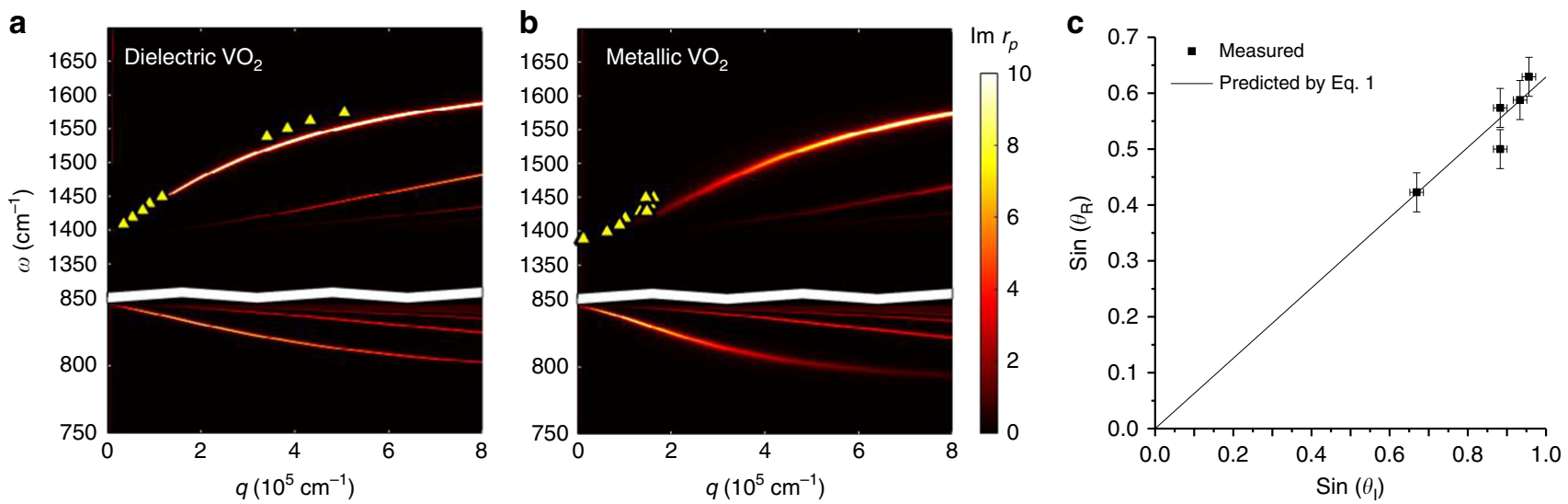

Fig. 3 Hyperbolic polariton dispersion in hBN over both dielectric (a) and metallic (b) domains of $\mathrm{VO}_{2}$ compared with numerical calculations. The horizontal white line shown in $\mathbf{a}$ and $\mathbf{b}$ indicates a break in the graph, between upper (1394-1650 $\left.\mathrm{cm}^{-1}\right)$ and lower $\left(785-845 \mathrm{~cm}^{-1}\right)$ Reststrahlen bands. From the measured dispersion, the angle of refracted waves at $1450 \mathrm{~cm}^{-1}$ can be computed for a given incident angle and compared against experimentally measured results in $\mathbf{c}$. There has been no fitting in this result. The $x$ and $y$ error bars in $\mathbf{c}$ represent the SD of measurements of incident $\left( \pm 1^{\circ}\right)$ and refracted $\left( \pm 2^{\circ}\right)$ angles, respectively
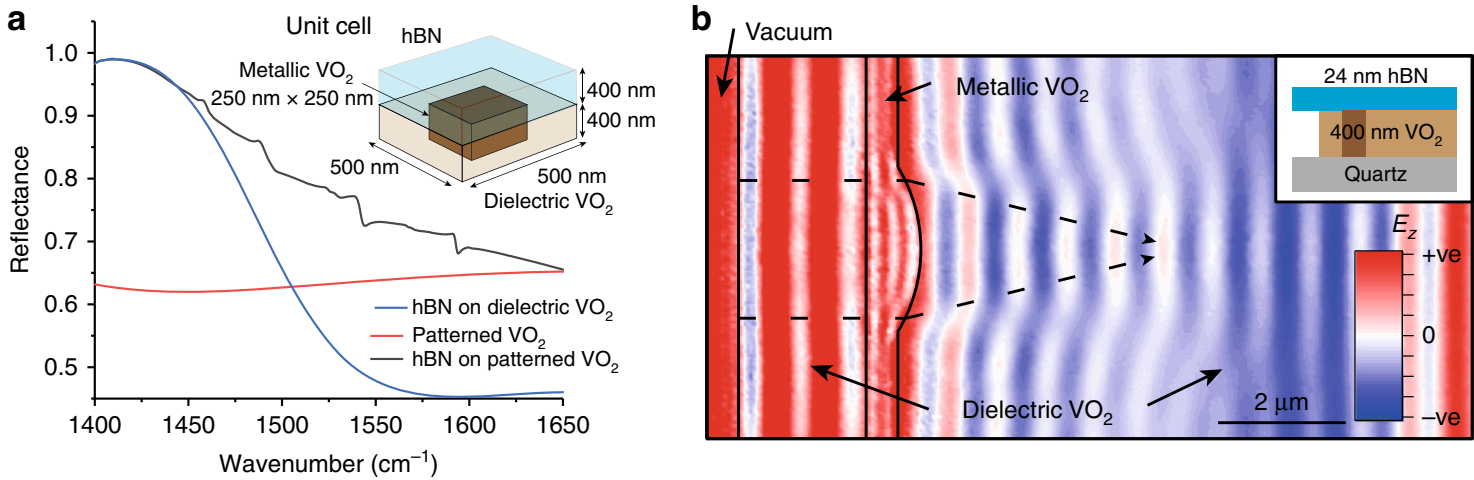

Fig. 4 Schematic of refractive planar optics and reconfigurable resonators using phase-change materials. a A tunable polariton metasurface of hBN and $\mathrm{VO}_{2}$, which consists of a continuous film of $\mathrm{hBN} 400 \mathrm{~nm}$ thick on top of $400 \mathrm{~nm}$ of $\mathrm{VO}_{2}$. By patterning the $\mathrm{VO}_{2}$ with metallic domains, we can excite a rewritable reflection profile, which cannot be generated from either of the materials alone. The pitch is $500 \mathrm{~nm}$, with a particle length $250 \mathrm{~nm}$. $\mathbf{b} \mathrm{A}$ simulation of a refractive polariton lens, which uses a semi-circular domain of metallic $\mathrm{VO}_{2}$ to launch polariton waves at $1418 \mathrm{~cm}^{-1}$

adherence to Snell's law for HPhPs (Fig. 3c). This result is consistent with numerical simulations at a range of different angles and frequencies (see Supplementary Note 4 and Supplementary Fig. 6) confirming that Snell's law holds for HPhPs propagating across domain boundaries. Systematic investigation of polariton propagation and refraction at multiple angles was not possible in prior work ${ }^{37}$ and thus the results presented here demonstrate that the tools and concepts of refractive optics are applicable in near-field optical designs as well. Indeed, the repeatable nature of both the change in polariton wavelength and Snell's law demonstrates that this platform can steer polariton propagation by proper design of the local dielectric environment.

Towards refractive near-field optics. The ability to control $\mathrm{HPhPs}$ propagating across phase-domain boundaries opens several possibilities for engineering lithography-free metasurfaces and near-field optics. As an example motivated by prior work ${ }^{12}$, we investigated the possibility for creating rewritable nanoresonators using this technique, where a periodic array of metallic square domains is created inside the $\mathrm{VO}_{2}$ crystal underneath the hBN (inset Fig. 4a). In Fig. 4a, we show the numerically calculated reflection spectrum from a hBN crystal on top of a dielectric $\mathrm{VO}_{2}$ (blue curve), $\mathrm{VO}_{2}$ patterned with metallic and dielectric domains (red curve), and hBN on top of such a patterned $\mathrm{VO}_{2}$ structure (black curve). In the simulated spectra for the hBN on top of patterned $\mathrm{VO}_{2}$, there are peaks corresponding to a series of $\mathrm{HPhP}$ modes. Although these peaks are relatively small in amplitude (as this geometry has not been optimized for an intense resonant response), these modes can be tuned in frequency by changing the width and periodicity of the metallic domain (see Supplementary Note 5 and Supplementary Fig. 7 for a complete discussion). Thus, in principle, by controlling the size and shape of the metallic domain, one can realize a resonant response that previously was only observed in nanofabricated structures of $\mathrm{hBN}^{20,39-41}$. This implies that such resonators can be realized without the additional induced losses that are incurred with most nanofabrication approaches ${ }^{42-44}$. Such resonators could also be achieved experimentally by doping to change the local phase transition properties of $\mathrm{VO}_{2}{ }^{45}$.

Refraction of HPhPs across boundaries also enlarges the toolbox for near-field optics to include those of conventional refractive systems, such as in-plane lenses, whereby polaritons are focused to a point via refraction. A simulation of such a lens is shown in Fig. $4 b$, where HPhPs are launched into hBN at the left crystal edge and propagate inward to a region over a hemispherical $\mathrm{VO}_{2}$ metallic domain, after which they are focused to a spot in the area over the dielectric $\mathrm{VO}_{2}$. Here, the combination of hyperbolic media and PCMs is critical, because 
for conventional surface polaritons, the high losses of the PCM metallic state would preclude polariton propagation and thus the polariton refraction required to induce focusing. Although experimentally we demonstrate the principle of this reconfigurable nano-optics platform using heterostructures comprising thin slabs of $\mathrm{hBN}$ on $\mathrm{VO}_{2}$ single crystals, this approach can readily be generalized to other materials. To demonstrate this, we have simulated a nanophotonic waveguide using both $\mathrm{VO}_{2}$ and $\mathrm{GeSbTe}^{12}$ as the underlying PCMs (see Supplementary Note 6 and Supplementary Fig. 8). The non-volatile nature of the phase change in $\mathrm{GeSbTe}^{12}$, where both states of the PCM are stable at room temperature, offers significant benefits for laser-writingbased approaches aimed at realizing complicated nanophotonic architectures. Although the device we present here is a conceptual prototype, our system could be realized in practice and scaled by using $\mathrm{VO}_{2}$ or GeSbTe films grown by sputtering and boron nitride grown by metal organic chemical vapor deposition ${ }^{46}$. There remain numerous material challenges-such as the growth of high-quality, large-area $\mathrm{hBN}$-in realizing such a system, but this provides a route to achieving scalable reconfigurable devices.

\section{Discussion}

We have experimentally demonstrated that the dispersion of HPhPs can be controlled using the permittivity changes inherent in the different phases of PCMs. This enables the direct launching, reflection, transmission, and refraction of HPhP waves at the domain boundaries between the different phases of the PCM, due to the large change in $\mathrm{HPhP}$ wavelength (here, by a factor of 1.6) that occurs for modes propagating in the $\mathrm{hBN}$ over each of these domains. Thermal cycling of the $\mathrm{hBN}-\mathrm{VO}_{2}$ heterostructure creates a range of domain-boundary geometries in the PCM, enabling the demonstration of various near-field phenomena. By inducing well-defined domain structures, it will be possible to design reconfigurable $\mathrm{HPhP}$ resonators and refractive optics in a planar, compact format at dimensions far below the diffraction limit. Beyond the implications for integrated nanophotonics, reconfigurable $\mathrm{HPhP}$ resonators could be used to match resonant frequencies to local molecular vibrational modes for the realization of dynamic surface-enhanced infrared absorption (SEIRA) spectroscopy ${ }^{41}$. Although in our case we have experimentally demonstrated these concepts using $\mathrm{hBN}$ on $\mathrm{VO}_{2}$, using different combinations of PCMs (such as GeSbTe) and other hyperbolic materials (such as transition metal oxides ${ }^{31}$ ) could see expanded applications over a wide frequency range. Ultimately, we anticipate that the combination of low-loss, hyperbolic materials, and latchable PCMs will result in applications in lithography-free design and fabrication of optical and optoelectronic devices, whereas volatile PCMs could be used for dynamic modulation of photonic structures.

\section{Methods}

Device fabrication. $\mathrm{VO}_{2}$ single crystals were grown by physical vapor transport in a quartz tube furnace at $810^{\circ} \mathrm{C}$ under 1.7 Torr $\mathrm{Ar}$ gas at a flow rate of 25 s.c.c.m. Vanadium pentoxide $\left(\mathrm{V}_{2} \mathrm{O}_{5}\right)$ powder $(\sim 0.3 \mathrm{~g}$, Sigma Aldrich 221899$)$ was placed in a quartz boat $(10 \times 1 \times 1 \mathrm{~cm})$ upstream of the desired substrates and heated for $1 \mathrm{~h}$. Evaporated $\mathrm{V}_{2} \mathrm{O}_{5}$ was reduced to $\mathrm{VO}_{2}$ in this process and deposited on quartz (0001) substrates. Representative crystals from each sample were investigated using Raman spectroscopy to identify the $\mathrm{VO}_{2}$ phase and optical microscopy to verify the thermal phase transition. Smaller, loose crystals located on the substrate surface were removed by adhesion to a heated $\left(60^{\circ} \mathrm{C}\right)$ layer of PMMA firmly brought into contact with the sample and subsequently retracted.

The isotopically enriched hBN crystals were grown from high-purity elemental ${ }^{10} \mathrm{~B}$ (99.22 at\%) powder by using the metal-flux method. A Ni-Cr-B powder mixture at respectively $48 \mathrm{wt} \%, 48 \mathrm{wt} \%$, and $4 \mathrm{wt} \%$ was loaded into an alumina crucible and placed in a single-zone furnace. The furnace was evacuated and then filled with $\mathrm{N}_{2}$ and forming gas ( $5 \%$ hydrogen in balance argon) to a constant pressure of 850 Torr. During the reaction process, the $\mathrm{N}_{2}$ and forming gases continuously flowed through the system with rates of 125 s.c.c.m. and 25 s.c.c.m., respectively. All the nitrogen in the hBN crystal originated from the flowing $\mathrm{N}_{2}$ gas.
The forming gas was used to minimize oxygen and carbon impurities in the hBN crystal. After a dwell time of $24 \mathrm{~h}$ at $1550^{\circ} \mathrm{C}$, the hBN crystals were precipitated onto the metal surface by cooling at a rate of $1{ }^{\circ} \mathrm{C} / \mathrm{h}$ to $1500{ }^{\circ} \mathrm{C}$, and then the system was quickly quenched to room temperature. Bulk crystals were exfoliated from the metal surface using thermal release tape. Crystals were subsequently mechanically exfoliated onto a PMMA/PMGI (polymethylglutarimide) polymer bilayer on silicon. Flakes were then transferred from the polymer substrate onto $\mathrm{VO}_{2}$ single crystals using a semi-dry technique and the polymer membrane was removed using acetone and isopropyl alcohol.

Numerical simulations. Numerical simulations were conducted in CST Studio Suite 2017 using the frequency domain solver with plane waves incident at $45^{\circ}$ and Floquet boundary conditions. In these simulations, polariton modes were only launched by scattering from edges in the simulation and field profiles were extracted using frequency monitors. All results used thicknesses consistent with that measured in topographic maps of the samples. Dielectric functions were taken from ref. ${ }^{18}$ for isotopically enriched $\mathrm{hBN}$, from ref. ${ }^{47}$ for $\mathrm{VO}_{2}$, and from ref. ${ }^{48}$ for GeSbTe.

sSNOM measurements. Near-field nano-imaging experiments were carried out in a commercial (www.neaspec.com) s-SNOM based around a tapping-mode atomic force microscope. A metal-coated Si-tip of apex radius $R \approx 20 \mathrm{~nm}$ that oscillates at a frequency of $\Omega \approx 280 \mathrm{kHz}$ and tapping amplitude of about $100 \mathrm{~nm}$ is illuminated by monochromatic quantum cascade laser laser beam at a wavelength $\lambda=6.9 \mu \mathrm{m}$ and at an angle $45^{\circ}$ to the sample surface. Scattered light launches hBN HPhPs in the device and the tip then re-scatters light (described more completely in the main text) for detection in the far-field. Background signals are efficiently suppressed by demodulating the detector signal at the second harmonic of the tip oscillation frequency and employing pseudo-heterodyne interferometric detection.

\section{Dataset availability statement}

The datasets generated during and/or analyzed during the current study are available from the corresponding authors upon reasonable request.

Received: 28 June 2018 Accepted: 27 September 2018

Published online: 22 October 2018

\section{References}

1. Yu, N. \& Capasso, F. Flat optics with designer metasurfaces. Nat. Mater. 13, 139-150 (2014).

2. Staude, I. \& Schilling, J. Metamaterial-inspired silicon nanophotonics. Nat. Photonics 11, 274-284 (2017).

3. Kildishev, A. V., Boltasseva, A. \& Shalaev, V. M. Planar photonics with metasurfaces. Science (Wash.) 339, 1232009 (2013).

4. Meinzer, N., Barnes, W. L. \& Hooper, I. R. Plasmonic meta-atoms and metasurfaces. Nat. Photonics 8, 889-898 (2014).

5. Khurgin Jacob, B. Relative merits of phononics vs. plasmonics: the energy balance approach. Nanophotonics 7, 305 (2018).

6. Wuttig, M., Bhaskaran, H. \& Taubner, T. Phase-change materials for nonvolatile photonic applications. Nat. Photonics 11, 465-476 (2017).

7. Yang, Z. \& Ramanathan, S. Breakthroughs in photonics 2014: phase change materials for photonics. IEEE Photonics J. 7, 1-5 (2015).

8. Abate, Y. et al. Control of plasmonic nanoantennas by reversible metalinsulator transition. Sci. Rep. 5, 8 (2015).

9. Liu, M., Sternbach, A. J. \& Basov, D. N. Nanoscale electrodynamics of strongly correlated quantum materials. Rep. Prog. Phys. 80, 27 (2017).

10. McGahan, C. et al. Geometric constraints on phase coexistence in vanadium dioxide single crystals. Nanotechnology 28, 085701-085701 (2017).

11. Qazilbash, M. M. et al. Mott transition in $\mathrm{VO}_{2}$ revealed by infrared spectroscopy and nano-imaging. Science 318, 1750 (2007).

12. Li, P. et al. Reversible optical switching of highly confined phonon-polaritons with an ultrathin phase-change material. Nat. Mater. 15, 870-875 (2016).

13. Yin, X. H. et al. Beam switching and bifocal zoom lensing using active plasmonic metasurfaces. Light Sci. Appl. 6, 7 (2017).

14. Karvounis, A., Gholipour, B., MacDonald, K. F. \& Zheludev, N. I. Alldielectric phase-change reconfigurable metasurface. Appl. Phys. Lett. 109, 051103 (2016).

15. Guo, P. J. et al. Conformal coating of a phase change material on ordered plasmonic nanorod arrays for broadband all-optical switching. Acs Nano 11, 693-701 (2017).

16. Kats, M. A. et al. Ultra-thin perfect absorber employing a tunable phase change material. Appl. Phys. Lett. 101, 221101 (2012).

17. Krishnamoorthy, H. N. S., Zhou, Y., Ramanathan, S., Narimanov, E. E. \& Menon, V. M. Tunable hyperbolic metamaterials utilizing phase chane heterostructures. Appl. Phys. Lett. 104, 121101 (2014). 
18. Giles, A. J. et al. Ultra-low-loss polaritons in isotopically pure boron nitride. Nat. Mater. 17, 134-139 (2017).

19. Vuong, T. Q. P. et al. Isotope engineering of van der Waals interactions in hexagonal boron nitride. Nat. Mater. 17, 152-158 (2018).

20. Caldwell, J. D. et al. Sub-diffractional, volume-confined polaritons in the natural hyperbolic material hexagonal boron nitride. Nat. Commun. 5, 522 (2014).

21. Dai, S. et al. Tunable phonon polaritons in atomically thin van der Waals crystals of boron nitride. Sci. (Wash.) 343, 1125-1129 (2014).

22. Korzeb, K., Gajc, M. \& Pawlak, D. A. Compendium of natural hyperbolic materials. Opt. Express 23, 25406-25424 (2015).

23. Low, T. et al. Polaritons in layered two-dimensional materials. Nat. Mater. 16, 182-194 (2017).

24. Sun, J., Litchinitser, N. M. \& Zhou, J. Indefinite by nature: from ultraviolet to terahertz. ACS Photonics 1, 293-303 (2014).

25. Duan, J. et al. Launching phonon polaritons by natural boron nitride wrinkles with modifiable dispersion by dielectric environments. Adv. Mater. 29, 1702494 (2017).

26. Poddubny, A., Iorsh, I., Belov, P. \& Kivshar, Y. Hyperbolic metamaterials. Nat. Photonics 7, 948-957 (2013).

27. Vakil, A. \& Engheta, N. Transformation optics using graphene. Sci. (Wash.) 332, 1291-1294 (2011).

28. Yang, X., Yao, J., Rho, J., Xiaobo, Y. \& Zhang, X. Experimental realization of three-dimensional indefinite cavities at the nanoscale with anomalous scaling laws. Nat. Photonics 6, 450-453 (2012).

29. Dai S., et al. Manipulation and steering of hyperbolic surface polaritons in hexagonal boron nitride. Adv. Mater. 30, 1706358 (2018).

30. Li, P. et al. Infrared hyperbolic metasurface based on nanostructured van der Waals materials. Science 359, 892-896 (2018).

31. Zebo, Z. et al. Highly confined and tunable hyperbolic phonon polaritons in van der Waals semiconducting transition metal oxides. Adv. Mater. 30, 1705318 (2018).

32. Fei, Z. et al. Gate-tuning of graphene plasmons revealed by infrared nanoimaging. Nature 487, 82-85 (2012).

33. Chen, J. et al. Optical nano-imaging of gate-tunable graphene plasmons. Nature 487, 77-81 (2012).

34. Dai, S. et al. Efficiency of launching highly confined polaritons by infrared light incident on a hyperbolic material. Nano. Lett. 17, 5285-5290 (2017).

35. Dai, S. et al. Subdiffractional focusing and guiding of polaritonic rays in a natural hyperbolic material. Nat. Commun. 6, 6963 (2015).

36. $\mathrm{Li}, \mathrm{P}$. et al. Hyperbolic phonon-polaritons in boron nitride for near-field optical imaging and focusing. Nat. Commun. 6, 7507 (2015)

37. Alonso-González, P. et al. Controlling graphene plasmons with resonant metal antennas and spatial conductivity patterns. Science 344, 1369-1373 (2014).

38. Yu, N. et al. Light propagation with phase discontinuities: generalized laws of reflection and refraction. Science 334, 333 (2011).

39. Brown, L. V. et al. Nanoscale mapping and spectroscopy of nonradiative hyperbolic modes in hexagonal boron nitride nanostructures. Nano. Lett. 18, 1628-1636 (2018).

40. Giles, A. J. et al. Imaging of anomalous internal reflections of hyperbolic phonon-polaritons in hexagonal boron nitride. Nano. Lett. 16, 3858-3865 (2016).

41. Autore M., et al. Boron nitride nanoresonators for phonon-enhanced molecular vibrational spectroscopy at the strong coupling limit. Light Sci. Appl. https://doi.org/10.1038/lsa.2017.172 (2017).

42. Caldwell, J. D. et al. Low-loss, extreme subdiffraction photon confinement via silicon carbide localized surface phonon polariton resonators. Nano. Lett. 13, 3690-3697 (2013).

43. Ocelic, N. \& Hillenbrand, R. Subwavelength-scale tailoring of surface phonon polaritons by focused ion-beam implantation. Nat. Mater. 3, 606-609 (2004).

44. Bassim, N. D., Giles, A. J., Ocola, L. E. \& Caldwell, J. D. Fabrication of phonon-based metamaterial structures using focused ion beam patterning. Appl. Phys. Lett. 112, 091101 (2018).
45. Chen, Z. et al. Self-assembled, nanostructured, tunable metamaterials via spinodal decomposition. ACS Nano 10, 10237-10244 (2016).

46. Takahashi, T., Itoh, H. \& Takeuchi, A. Chemical vapor deposition of hexagonal boron nitride thick film on iron. J. Cryst. Growth 47, 245-250 (1979).

47. Kats M. A. Optics at interfaces: ultra-thin color coatings, perfect absorbers, and metasurfaces Ph.D. thesis, Harvard University (2014).

48. Kremers S. Optical properties of phase change materials for novel optical and electrical storage applications Ph.D. thesis, RWTH Aachen (2009).

\section{Acknowledgements}

We thank Professor Misha Fogler for providing a script to calculate the dispersion of HPhPs. T.G.F. and S.T.W. thank the staff of the Vanderbilt Institute for Nanoscience (VINSE) for technical support during fabrication and Kiril Bolotin for preliminary design of the $2 \mathrm{D}$ transfer tool used. Support for the ${ }^{10} \mathrm{~B}$-enriched hBN crystal growth was provided by the National Science Foundation, grant number CMMI 1538127. Y.A. and N.A. gratefully acknowledge support provided by the Air Force Office of Scientific Research (AFOSR) grant number FA9559-16-1- 0172. The work of A.F. is supported by the National Science Foundation grant 1553251

\section{Author contributions}

R.F.H, Y.A., and J.D.C. conceived and guided the experiments. S.T.W. grew the $\mathrm{VO}_{2}$ crystals and identified the phase domains. S.L. and J.H.E. grew the hBN crystals. T.G.F. and S.T.W. fabricated the $\mathrm{hBN}-\mathrm{VO}_{2}$ heterostructure. A.F. and N.A. performed s-SNOM mapping experiments of the sample at various temperatures and incident frequencies. $\mathrm{T}$. G.F. advised on experimental questions, developed the electromagnetic models, and analyzed s-SNOM data to show the presence of refraction. T.G.F. also conducted electromagnetic simulations of resonators, lenses, and waveguides. S.T.W. and J.R.M. analyzed s-SNOM data and calculated the dispersion curves. All authors contributed to writing the manuscript.

\section{Additional information}

Supplementary Information accompanies this paper at https://doi.org/10.1038/s41467018-06858-y.

Competing interests: The authors declare no competing interests.

Reprints and permission information is available online at http://npg.nature.com/ reprintsandpermissions/

Publisher's note: Springer Nature remains neutral with regard to jurisdictional claims in published maps and institutional affiliations.

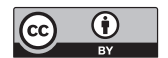

Open Access This article is licensed under a Creative Commons Attribution 4.0 International License, which permits use, sharing, adaptation, distribution and reproduction in any medium or format, as long as you give appropriate credit to the original author(s) and the source, provide a link to the Creative Commons license, and indicate if changes were made. The images or other third party material in this article are included in the article's Creative Commons license, unles indicated otherwise in a credit line to the material. If material is not included in the article's Creative Commons license and your intended use is not permitted by statutory regulation or exceeds the permitted use, you will need to obtain permission directly from the copyright holder. To view a copy of this license, visit http://creativecommons.org/ licenses/by/4.0/.

(C) The Author(s) 2018 